\title{
ANALYTIC TENSOR AND ITS GENERALIZATION
}

\author{
SHUN-ICHI TACHIBANA
}

(Received September 20, 1959)

In our previous papers [7], [8], the notion of almost-analytic vector was introduced in certain almost-Hermitian spaces. In this paper we shall deal with tensors and obtain the notion of $\Phi$-tensors which contains, as special cases, the one of analytic tensors and decomposable tensors.

1. Let us consider an $n$-dimensional space $^{2)}$ which admits a tensor field $\boldsymbol{\varphi}_{i}{ }^{j}$ of type $(1,1)$. Let $\xi_{(i)}{ }^{(j)} \equiv \xi_{i_{p}} \cdots i_{1}{ }^{\prime}{ }^{\prime} \cdots j_{1}$ be a tensor of type $(q, p)$. If it commutes with $\boldsymbol{\varphi}_{i}{ }^{j}$, then we shall say that $\xi_{(i)}{ }^{(j)}$ is pure with respect to the corrresponding indices, namely it is pure with respect to $i_{k}$ and $j_{h}$, if

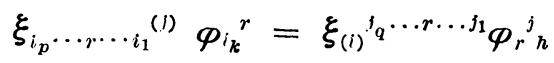

and pure with respect to $i_{k}$ and $i_{h}$, if

$$
\xi_{i_{p} \cdot \cdots+\cdots i_{k} \cdots i_{1}}{ }^{(j)} \boldsymbol{\varphi}_{i_{k}}{ }^{r}=\xi_{i_{p} \cdots i_{k} \cdots \cdots i_{1}}{ }^{(j)} \boldsymbol{\phi}_{i_{h}}{ }^{r} .
$$

If $\xi_{(i)}{ }^{(j)}$ anti-commutes with $\varphi_{i}{ }^{j}$ then we shall say that it is hybrid with respect to the corresponding indices. Thus if

$$
\xi_{i_{p} \ldots r \cdots i_{1}{ }^{(j)}} \boldsymbol{\varphi}_{i_{k}}{ }^{r}=-\xi_{(i)}{ }^{j q \ldots r \cdots j_{1}} \boldsymbol{\varphi}_{r}^{j_{k}},
$$

for example, holds good, then it is hybrid with respect to $i_{k}$ and $j_{h}$. $\xi_{(i)}^{(j)}$ is called pure (resp. hybrid) if it is pure (resp. hybrid) with respect to all its indices.

$\boldsymbol{\phi}_{i}{ }^{j}$ itself and $\delta_{i}{ }^{j}$ are examples of the pure tensor. If $\boldsymbol{\phi}_{i}{ }^{j}$ is a regular tensor i.e. $\operatorname{det}\left(\varphi_{i}^{j}\right) \neq 0$, then the tensor whose components are given by the elements of the inverse matrix of $\left(\varphi_{i}{ }^{j}\right)$ is also pure.

LEMMA 1. If $\xi_{(i)}^{(i)}$ is pure (hybrid) with respect to some indices, then so is $\xi_{(i)}^{*}{ }^{(i)}=\xi_{i_{p}} \ldots i_{2},{ }^{\left({ }^{(j)}\right.} \boldsymbol{\phi}_{i_{1}}{ }^{r}$.

We shall prove only the case when $\xi_{(i)}^{(j)}$ is pure with respect to $i_{1}$ and $i_{k}(k \neq 1)$. In fact, we have

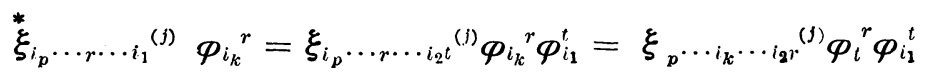

1) The number in backets refers to the bibliography at the end of the paper.

2) We shall mean by a space a differentiable manifold of class $C^{\infty}$, and denote by $x^{i}$ its local coordinates. Indices run over $1,2, \cdots \cdots n$. 


$$
=\stackrel{*}{\xi}_{i_{p} \cdots i_{2} t}^{(j)} \rho_{i_{1}}{ }^{t} \quad \text { q.e.d. }
$$

LEMMA 2. If a skew-synmetric tensor $\xi_{(i)}$ is pure, then $\stackrel{*}{\xi}_{(i)}=\xi_{i_{p} \cdots i_{2 r}} \boldsymbol{\varphi}_{i_{1}}{ }^{r}$ is also a skew-symmetric pure tensor.

In fact, $\stackrel{*}{\xi}_{(i)}$ is pure by virtue of Lemma 1 . It is evident that it is skewsymmetric with respect to $i_{k}$ and $i_{h}(k, h \neq 1)$. For $k \neq 1$, we have

$$
\begin{aligned}
& {\stackrel{*}{\xi_{p} \cdots i_{k} \cdots i_{1}}}^{*}=\xi_{i_{p} \cdots i_{k} \cdots i_{2} \gamma} \boldsymbol{\varphi}_{i_{1}}{ }^{r}=\xi_{i_{p} \cdots \cdots i_{1}} \boldsymbol{\varphi}_{i_{k}}{ }^{r}
\end{aligned}
$$

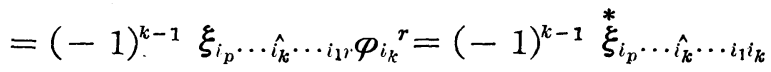

$$
\begin{aligned}
& =-{\stackrel{\xi}{*} i_{p} \cdots i_{1} \cdots i_{2} i_{k} \text {. }}^{*} \text { q. e. d. }
\end{aligned}
$$

If $\xi_{(i)}{ }^{t(1)} \equiv \xi_{(i)}{ }^{i j_{q} \cdots j_{1}}$ is a pure tensor of type $(q+1, p)$, then $u_{t} \xi_{(i)}^{t(j)}$ is also pure for a (covariant) vector $u_{i}$. Generalizing this fact, we have easily

LEMMA 3. Let $\xi_{(i)}^{\left({ }^{(j)}\right.}$ and $\eta_{(a)}{ }^{t(b)}$ be pure tensors of type $(q, p+1)$ and $\left(q^{\prime}+1, p^{\prime}\right)$ respectively. Then $\xi_{t(i)}^{(1)} \eta_{(a)}{ }^{(b)}$ is also a pure tensor of type $(q+$ $\left.q^{\prime}, p+p^{\prime}\right)$, provided that $p+p^{\prime} \neq 0$ or $q+q^{\prime} \neq 0$.

A tensor $\phi_{i}{ }^{j}$ is called an almost-product structure, if it satisfies $\varphi_{i}{ }^{r} \phi_{r}{ }^{3}=$ $\delta_{i}{ }^{j}$, and is called an almost-complex structure, if it satisfies $\varphi_{i}{ }^{r} \varphi_{r}{ }^{j}=-\delta_{i}{ }^{j}$, [1], [2], [4], [12].

In these cases, we can verify the following lemmas.

LEMMA 4. Let $\varphi_{i}{ }^{j}$ be a tensor such that $\varphi_{i}{ }^{r} \varphi_{r}{ }^{j}=\varepsilon \delta_{i}{ }^{j}{ }^{3)}$ Then we have $\xi_{r}{ }^{r}=0$ for a hybrid tensor $\xi_{i}{ }^{j}$.

LEMMA 5. Let $\phi_{i}{ }^{j}$ be a tensor such that $\phi_{i}{ }^{r} \varphi_{r}{ }^{j}=\varepsilon \delta_{i}{ }^{j}$. If $\xi_{i}{ }^{j}\left(\xi^{i j}\right)$ is pure and $\eta_{j}^{\prime}\left(\eta_{i j}\right)$ is hybrid, then we have

$$
\xi_{i}{ }^{j} \eta_{j}{ }^{i}=0, \quad\left(\xi^{i j} \eta_{i j}=0\right) .
$$

LEMMA 6. Let $\phi_{i}{ }^{j}$ be a regular tensor, i.e. $\operatorname{rank}\left(\phi_{i}{ }^{j}\right)=n$. If $\xi_{k j i}\left(\xi_{k j}{ }^{i}\right)$ is hybrid, then it is a zero tensor.

In fact, we have

$$
\xi_{k r i} \varphi_{j}^{r}=-\xi_{r j i} \varphi_{k i}^{r}=\xi_{k j r} \varphi_{i}^{r}=-\xi_{k r i} \varphi_{j}^{r},
$$

from which we find $\xi_{k j i}=0$.

q. e. d.

Now consider an almost-complex structure $\phi_{i}{ }^{j}$, then if we choose a suitable frame at a point, $\varphi_{i}{ }^{j}$ has the following components at the point.

$$
\phi_{i}^{\beta}=i \delta_{\alpha}{ }^{\beta}, \quad \phi_{\bar{\alpha}}^{\bar{\alpha}^{\bar{\beta}}}=-i \delta_{\bar{\alpha}}^{\bar{\beta}}, \quad \phi_{\bar{\alpha}}{ }^{\beta}=\phi_{\alpha^{\beta}}{ }^{\bar{\beta}}=0 .{ }^{4)}
$$

3) In this paper, by $\varepsilon$ we shall always mean \pm 1 .

4) Indices $a, \beta$, run over $1, \ldots, m(=n / 2)$ and $\bar{\alpha}=m+\alpha$. 

ations

With respect to this frame, the equation (1) is equivalent to the equ-

$$
\xi_{i_{p} \cdots \alpha_{k} \cdots i_{1}}{ }^{j_{q} \cdots \bar{\rho}_{h} \cdots j_{1}}=0, \quad \xi_{i_{p} \cdots \bar{\alpha}_{k} \cdots i_{1}{ }^{j_{q}} \cdots \beta_{h} \cdots j_{1}}=0,
$$

and the equation (2) is equivalent to

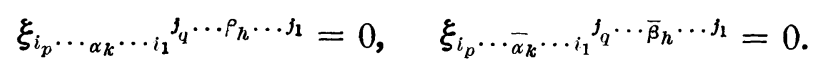

In this sense we have used the terminologies "pure" and "hybrid" [6], [11].

2. An almost-Hermitian space admits, by definition, a Riemannian metric tensor $g_{j i}$ and an almost-complex structure $\boldsymbol{\varphi}_{i}{ }^{j}$ such that $g_{r s} \boldsymbol{\varphi}_{j}{ }^{r} \boldsymbol{\varphi}_{i}{ }^{s}=g_{j i}$.

A Kählerian space is an almost-Hermitian one such that the equation

$$
\nabla_{l} \boldsymbol{\varphi}_{i}{ }^{n}=0
$$

is valid, where $\nabla_{l}$ denotes the operator of the covariant derivative with respect to the Christoffel's symbol $\left\{\begin{array}{c}h \\ j i\end{array}\right\}$.

We shall devote this section to a Kählerian space.

A pure tensor $\xi_{(i)}^{(j)}$ is called analytic [6], if its covariant derivative $\nabla_{l} \xi_{(i)}^{(j)}$ is also pure, i.e. it satisfies

$$
\begin{aligned}
& \varphi_{l}{ }^{r} \nabla r \xi_{(i)}{ }^{(j)}=\varphi_{i_{1}}{ }^{r} \nabla_{l} \xi_{i_{p}} \ldots i_{2} r{ }^{(i)}, \\
& \varphi_{l}^{r} \nabla r \xi_{(i)}^{(j)}=\varphi_{r}{ }^{j 1} \nabla_{l} \xi_{(i)}{ }^{{ }^{j}}{ } \cdots j_{2 r} .
\end{aligned}
$$

In fact, the equation (4) is equivalent to the following one with respect to complex coordinates $\left(z^{\alpha}, z^{\bar{\alpha}}\right)$ :

$$
\frac{\partial}{\partial z^{\bar{\lambda}}} \xi_{(\alpha)}^{(\beta)}=0, \quad \frac{\partial}{\partial z^{\lambda}} \xi_{(\bar{\alpha})}^{(\bar{\beta})}=0 .
$$

The definition (4) of the analytic tensor contains the Kählerian metric in appearence, but (5) is independent to the metric. Hence it is natural to ask if the notion of the analytic tensor is defined in a complex manifold with respect to real coordinates.

In this point of view, we shall attempt to eliminate the Christoffel's symbols in (4) by making use of (3).

If we write down (4) explicitly, we hvae

$$
\begin{aligned}
& \boldsymbol{\varphi}_{l}^{r}\left[\partial_{r} \xi_{(i)}^{(j)}+\sum_{k=1}^{q}\left\{\begin{array}{l}
j_{k} \\
r t
\end{array}\right\} \xi_{(i)}^{j}{ }^{j} \cdots \cdots j_{1}-\sum_{k=1}^{p}\left\{\begin{array}{c}
t \\
r i_{k}
\end{array}\right\} \xi_{i_{p} \cdots \omega_{i}{ }^{(j)}}\right] \\
& =\varphi_{i_{1}}{ }^{t}\left[\partial_{l} \xi_{i_{p} \cdots i_{2}}{ }^{(j)}+\sum_{k=1}^{q}\left\{\begin{array}{l}
j_{k} \\
l r
\end{array}\right\} \xi^{i_{p} \ldots i_{2} t}{ }^{j}{ }^{j} \cdots r \cdots j_{1}\right. \\
& \left.-\sum_{k=2}^{p}\left\{\begin{array}{c}
r \\
l i_{k}
\end{array}\right\} \xi_{i_{p} \cdots \cdots i_{\mathbf{g}} t^{(j)}}-\left\{\begin{array}{l}
r \\
l t
\end{array}\right\} \xi_{i_{p} \cdots i_{2} r}{ }^{(j)}\right],
\end{aligned}
$$


where we have put $\partial_{r}=\partial / \partial x^{r}$.

On the other hand, on taking account of (3), we have

$$
\begin{aligned}
\partial_{l} \boldsymbol{\varphi}_{t}{ }^{j_{k}}-\partial_{l} \boldsymbol{\varphi}_{l}{ }^{j_{k}} & =\left\{\begin{array}{c}
j_{k} \\
t r
\end{array}\right\} \boldsymbol{\varphi}_{l}{ }^{r}-\left\{\begin{array}{l}
j_{k} \\
l r
\end{array}\right\} \boldsymbol{\varphi}_{t}{ }^{r}, \\
\partial_{i_{1}} \boldsymbol{\varphi}_{l}{ }^{t}-\partial_{l} \boldsymbol{\varphi}_{i_{1}}{ }^{t} & =\left\{\begin{array}{c}
t \\
l r
\end{array}\right\} \boldsymbol{\varphi}_{i_{1}}{ }^{r}-\left\{\begin{array}{c}
t \\
i_{1} r
\end{array}\right\} \boldsymbol{\varphi}_{l}{ }^{r}, \\
\partial_{i_{k}} \boldsymbol{\varphi}_{l}{ }^{t} & =\left\{\begin{array}{c}
r \\
i_{k} l
\end{array}\right\} \boldsymbol{\varphi}_{r}{ }^{t}-\left\{\begin{array}{c}
t \\
i_{k} r
\end{array}\right\} \boldsymbol{\varphi}_{l}{ }^{r} .
\end{aligned}
$$

If we substitute these equations into (6) and take account of the purity of $\xi_{(i)}^{(j)}$, then we find

$$
\begin{aligned}
\boldsymbol{\varphi}_{l}{ }^{r} \partial_{r} \xi_{(i)}{ }^{(j)} & -\partial_{l} \stackrel{\xi}{(i)}^{(j)}+\sum_{k=1}^{p}\left(\partial_{i_{k}} \boldsymbol{\varphi}_{l}{ }^{r}\right) \xi_{i_{j} \cdots \cdots i_{1}}{ }^{(j)} \\
& +\sum_{k=1}^{q}\left(\partial_{l} \boldsymbol{\varphi}_{r}{ }^{i_{k}}-\partial_{r} \boldsymbol{\varphi}_{l}{ }^{j_{k}}\right) \xi_{(i)}{ }^{j_{q} \cdots r \cdots s_{1}}=0,
\end{aligned}
$$

where $\stackrel{*}{\xi}_{(i)}^{(1)}$ is defined by

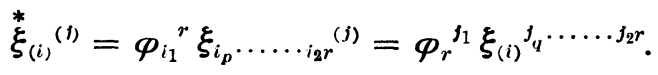

3. As we have known in the preceding section, the equation (7) which defines the analytic tensor in a Kählerian space does not contain the Kählerian metric. Following to this fact, we shall introduce an operator in a space which admits a tensor field of type $(1,1)$. The operator will produce from a pure tensor of type $(q, p)$ a new tensor of type $(q, p+1)$.

Let $\varphi_{i}{ }^{j}$ be a tensor of type $(1,1)$ and $\xi_{(i)}{ }^{(j)}$ a pure tensor of type $(q, p)$. Now we define an operator $\Phi$ by

$$
\begin{aligned}
& \Phi_{l} \xi_{(i)}{ }^{(l)}=\varphi_{l}{ }^{r} \partial_{r} \xi_{(i)}^{(j)}-\partial_{l} \stackrel{\xi}{(i)}^{(j)}+\sum_{k=1}^{p}\left(\partial_{i_{k}} \varphi_{l}{ }^{r}\right) \xi_{i_{p} \cdots r i_{1}}{ }^{(l)} \\
& +\sum_{k=1}^{q}\left(\partial_{l} \boldsymbol{\varphi}_{r}^{j_{k}}-\partial_{r} \boldsymbol{\varphi}_{l}^{j_{k}}\right) \xi_{(i)}{ }^{j}{ }^{2} \ldots . \ldots j_{1}
\end{aligned}
$$

where $\stackrel{*}{\xi}_{(i)}^{(i)}$ is given by (8).

In the rest of the present section, we shall show that $\Phi_{l} \xi_{(i)}^{(i)}$ is a tensor, if $\xi_{(i)}^{(j)}$ is pure.

Let $\Gamma_{j i}^{\prime}$ be an affine connection, $S_{j i}{ }^{n}$ its torsion tensor, i.e. $S_{j i}{ }^{n}=(1 / 2)$ $\left(\Gamma_{j i}^{h}-\Gamma_{i j}^{h}\right)$ and by $\nabla_{k}$ we shall denote the operator of covariant derivative with respect to $\Gamma_{j i}^{h}$. Hence if $v^{i}$ is a vector field, then its covariant derivative is given by $\nabla_{k} v^{i}=\partial_{k} v^{i}+\Gamma_{k r}^{i} v^{r}$.

If we represent (9) by terms of covariant derivatives, $\Phi_{l} \xi_{(i)}^{(j)}$ is the sum of the following five terms $a_{1}, \ldots \ldots, a_{5}$ : 


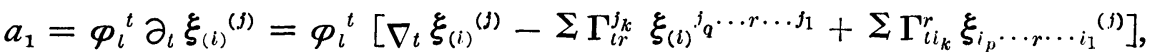

$$
\begin{aligned}
& a_{2}=-\partial_{l} \stackrel{*}{\xi}_{(i)}^{(j)}=-\nabla_{l} \stackrel{*}{\xi}_{(i)}^{(j)}+\Sigma \Gamma_{i t}^{j_{k}{ }^{k} \stackrel{*}{\xi}_{(i)}}{ }^{j_{1} \cdots t \cdots j_{1}}-\Sigma \Gamma_{l i_{k}}^{t} \stackrel{*}{\xi}_{i_{p} \cdots i_{i} i_{1}}^{(j)} \text {, }
\end{aligned}
$$

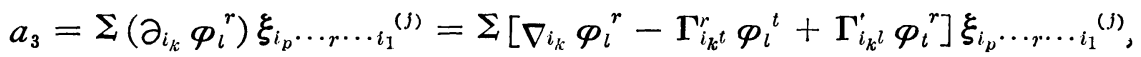

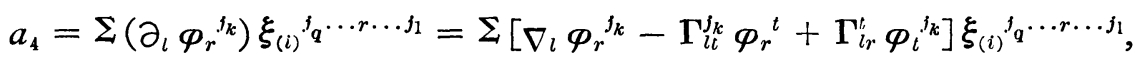

$$
\begin{aligned}
& a_{5}=-\left(\partial_{r} \varphi_{l}^{j_{k}}\right) \xi_{(i)}{ }^{\prime} q^{\cdots r \cdots j_{1}}=\Sigma\left[-\nabla_{r} \varphi_{l}^{j_{k}}+\Gamma_{r t}^{j_{k}} \varphi_{l}{ }^{t}-\Gamma_{r l} \varphi_{t}^{j_{k}}\right] \xi_{\left(l_{1}\right)}{ }^{j_{1} \cdots r \cdots j_{1}} \text {. }
\end{aligned}
$$

If we denote the $\lambda$-th term of $a_{\mu}$ by $a_{\mu \lambda}$, the following relations hold.

$$
\begin{aligned}
& a_{12}+a_{52}=2 \sum S_{r t}{ }^{j_{k}} \boldsymbol{\phi}_{l}{ }^{t} \xi_{(i)}{ }^{j}{ }^{j} \cdots \cdot \cdots j_{1}, \\
& a_{13}+a_{32}=2 \Sigma S_{t i_{k}}{ }^{r} \boldsymbol{\varphi}_{l}{ }^{t} \xi_{i_{p} \cdots r_{1} \cdots i_{1}}{ }^{(j)} \text {, } \\
& a_{43}+a_{53}=2 \Sigma S_{l r}{ }^{t} \boldsymbol{\varphi}_{t}{ }^{j_{k}} \xi_{(i)}{ }^{j}{ }^{j} \cdots r \cdots j_{1}, \\
& a_{22}+a_{42}=0 \text {, } \\
& a_{23}+a_{33}=2 \Sigma S_{i_{h} l}{ }^{t} \phi_{t}{ }^{r} \xi_{i_{p} \cdots r \cdots i_{1}}{ }^{(j)} .
\end{aligned}
$$

Thus we find that

$$
\begin{aligned}
& \Phi_{l} \xi_{(i)}^{-(j)}=\phi_{l}^{r} \nabla_{r} \xi_{(i)}^{(j)}-\nabla_{l} \xi_{(i)}^{*}(j) \\
& +\sum_{k=1}^{p}\left\{\nabla_{i_{k}} \boldsymbol{\varphi}_{l}{ }^{r}+2\left(S_{i_{k} l}{ }^{t} \boldsymbol{\varphi}_{t}^{r}-S_{i_{k} t}^{r} \boldsymbol{\varphi}_{l}{ }^{t}\right)\right\} \xi_{i_{p} \cdots i_{1}}{ }^{(j)}
\end{aligned}
$$

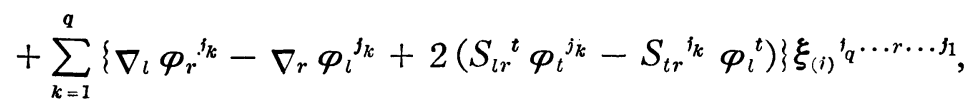

which shows that $\Phi_{l} \xi_{(i)}^{(i)}$ is a tensor.

4. In this section, we shall represent (9) in different forms. Using the notation in $\S 3$, we have

$$
\begin{aligned}
& a_{3}=\Sigma\left(\partial_{i_{k}}{\stackrel{*}{\xi_{i} \cdots l \cdots i_{1}}}^{(j)}-\varphi_{l}{ }^{r} \partial_{i_{k}} \xi_{i_{p} \cdots r \cdots i_{1}}{ }^{(j)}\right), \\
& a_{4}=q \partial_{l}{\stackrel{\xi}{\xi_{(i)}}}^{(j)}-\Sigma \varphi_{r^{\prime} k} \partial_{l} \xi_{(i)}{ }^{j} \cdots r \cdots j_{1} .
\end{aligned}
$$

Now if we put

$$
\partial_{<l} \xi_{(i)>}^{(i)}=\partial_{l} \xi_{(i)}^{(j)}-\sum_{k=1}^{p} \partial_{i, v} \xi_{i_{p} \ldots l \cdots i_{1}}{ }^{(i)},
$$

then, substituting (11) into (9), we find that

$$
\Phi_{l} \xi_{(i)}^{(i)}=\varphi_{l}{ }^{r} \partial_{<r} \xi_{(i)>}^{(j)}-\partial_{<l}{ }^{*} \xi_{(i)>}^{(j)}+\sum_{k=1}^{q}\left(\partial_{l} \varphi_{r}^{j_{k}}-\partial_{r} \varphi_{l}{ }^{j_{k}}\right) \xi_{(i)}{ }^{j_{g}} \cdots r \cdots s_{1}
$$

Hence if $\xi_{(i)}$ is a pure tensor of type $(0, p)$, it holds that 


$$
\Phi_{l} \xi_{(i)}=\varphi_{l}^{r} \partial_{<r} \xi_{(i)>}-\partial_{<l} \stackrel{*}{\xi}_{(i)>}
$$

In the next place, if we substitute (12) into (13), then we get

$$
\begin{aligned}
\Phi_{l} \xi_{(i)}^{(j)} & =\phi_{l}^{r} \partial_{<r} \xi_{(i)>}^{(j)}-\partial_{<l}{\stackrel{*}{\xi_{(i)}}}^{(j)}+q \partial_{l} \stackrel{*}{\xi}_{(i)}^{(j)} \\
& -\sum_{k=1}^{q}\left(\xi_{(i)}^{j}{ }^{j} \cdots \cdots j_{1} \partial_{r} \phi_{l}^{j_{k}}+\phi_{r}^{j_{k}} \partial_{l} \xi_{(i)}^{j} q^{j \cdots \cdots s_{1}}\right) .
\end{aligned}
$$

Hence if $\xi^{(j)}$ is a pure tensor of type $(q, 0)$, then we have

$$
\begin{aligned}
\Phi_{l} \xi^{(j)}=\phi_{l}^{r} \partial_{r} \xi^{(\jmath)} & +(q-1) \partial_{l} \xi^{(j)} \\
& -\sum_{k=1}^{q}\left(\xi^{\prime} q \cdots \cdots \nu_{1} \partial_{r} \varphi_{l j_{k}}+\varphi_{r}^{j_{k}} \partial_{l} \xi^{\xi_{q} \cdots \cdots j_{1}}\right),
\end{aligned}
$$

from which, in the case when $q=1$, we find

$$
\Phi_{l} \xi^{j}=-\left(\xi^{r} \partial_{r} \phi_{l}{ }^{j}-\phi_{l}{ }^{r} \partial_{r} \xi^{j}+\phi_{r}{ }^{j} \partial_{l} \xi^{r}\right)=-\underset{\xi}{\dot{f}} \phi_{l}{ }^{j},
$$

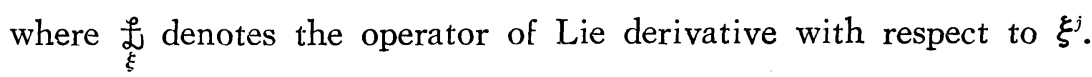

If $\xi_{i}{ }^{j}$ is a pure tensor of type $(1,1)$, then we have from (9)

$$
\Phi_{l} \xi_{i}{ }^{j}=\varphi_{l}{ }^{r} \partial_{r} \xi_{i}{ }^{j}-\varphi_{r}{ }^{j} \partial_{l} \xi_{i}{ }^{r}+\xi_{r}{ }^{j} \partial_{i} \varphi_{l}{ }^{r}-\xi_{i}{ }^{r} \partial_{r} \varphi_{l}{ }^{j},
$$

which is nothing but $\sum^{1,2} \iota^{j}{ }^{j}$ in Nijenhuis' paper [3].

In particular, we have $\Phi_{l} \delta_{i}{ }^{j}=0$.

5. Let $\xi_{t(i)}^{(j)} \equiv \xi_{i_{p} \ldots i_{1}}^{(j)}$ and $\eta_{(a)^{t(b)}}{ }^{(j)} \eta_{(a)}{ }^{t b} q^{\prime} \ldots b_{1}$ be pure tensors of type $(q, p+1)$ and type $\left(q^{\prime}+1, p^{\prime}\right)$ respectively. Then we shall verify the following formula :

$$
\Phi_{l}\left(\xi_{l(i)}{ }^{(j)} \eta_{(a)^{t(b)}}\right)=\left(\Phi_{l} \xi_{l(i)}^{(j)}\right) \eta_{(a)^{t(b)}}+\xi_{l(l)}^{(j)} \Phi_{l} \eta_{(a)}{ }^{t(b)},
$$

if $p+p^{\prime} \neq 0$ or $q+q^{\prime} \neq 0$.

In fact, the left hand side is the sum of the following six terms $b_{1}$, $b_{6}$.

$$
\begin{aligned}
& b_{1}=\phi_{l}^{r} \partial_{r}\left(\xi_{l(i)}^{(i)} \eta_{(a)}{ }^{l(b)}\right), \\
& b_{2}=-\eta_{(a)}{ }^{l(b)} \partial_{l} \stackrel{*}{\xi}_{t(i)}^{(j)}-\xi_{t(l)}{ }^{(j)} \partial_{l}{ }^{*}(a){ }_{(a)}{ }^{(b)}+\xi_{t(l)}{ }^{(j)} \eta_{(a)}{ }^{r(b)} \partial_{l} \varphi_{r}{ }^{t}, \\
& b_{3}=\Sigma\left(\partial_{i_{k}} \boldsymbol{\phi}_{l}^{r}\right) \xi_{t i_{p} \cdots \cdots_{i}{ }^{(j)}} \boldsymbol{\eta}_{(a)^{(t)}}{ }^{t(b)} \text {, } \\
& b_{4}=\Sigma\left(\partial_{a_{k}} \boldsymbol{\varphi}_{l}^{r}\right) \xi_{t(i)}{ }^{(j)} \eta_{a_{p^{\prime}}} \cdots \cdots \cdots a_{1}{ }^{(b)} \text {, }
\end{aligned}
$$

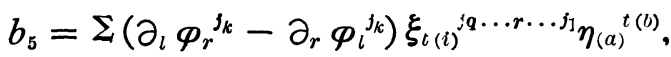




$$
b_{6}=\Sigma\left(\partial_{l} \varphi_{r}^{b_{k}}-\partial_{r} \boldsymbol{\varphi}_{l}^{b_{k}}\right) \xi_{l(i)}{ }^{(j)} \eta_{(a)}{ }^{t{ }^{\prime}}{ }^{\prime} \cdots r \cdots b_{1},
$$

from which we can easily obtain (14).

If a pure tensor (or a vecter) $\xi$ satisfes $\Phi_{l} \xi=0$, then we shall say that it is a $\Phi$-tensor (or $\Phi$-vector). If the tensor $\boldsymbol{\varphi}_{i}{ }^{j}$ is a complex structure, then a $\Phi$-tensor is an analytic tensor. If $\varphi_{i}{ }^{j}$ is a product structure i.e. an almostproduct structure such that its Nijenhuis' tensor vanishes, then a $\Phi$-tensor is decomposable.

From (14) we have

THEOREM 1. If $\xi_{t(i)}^{(j)}$ and $\eta_{(a)}{ }^{t(b)}$ are $\Phi$-tensors, then so is $\xi_{t(i)}^{(j)} \eta_{(a)}^{t(b)}$ provided that it is not a scalar.

6. Let us consider two Riemannian metrics $g_{j i}$ and $\boldsymbol{\phi}_{j i}$ which are not necessarily positive definite. Putting $\boldsymbol{\phi}_{i}{ }^{j}=\varphi_{i r} g^{\text {r. }}$ we shall introduce the operator $\Phi$ which is associated to $\varphi_{i}{ }^{j}$.

Since it holds that $g_{r i} \boldsymbol{\varphi}_{j}{ }^{r}=\boldsymbol{\varphi}_{j \ell}=\boldsymbol{\varphi}_{i j}=g_{j r} \boldsymbol{\varphi}_{i}{ }^{r}$, we know that $g_{j i}$ is pure. Taking account of $\stackrel{*}{g}_{j i}=\phi_{\text {ji }}$, we obtain

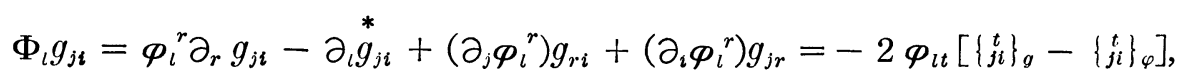

where $\left\{j_{i}^{t}\right\}_{g}$ and $\left\{j_{j i}^{t}\right\}_{\varphi}$ are the Christoffel's symbols formed by $g_{j i}$ and $\boldsymbol{\phi}_{j i}$ respectively. Thus we have

THEOREM 2. Let $g_{j i}$ and $\boldsymbol{\varphi}_{j i}$ be two Riemannian metrics. Then a necessary and sufficient condition in order that the Christoffel's symbols coincide with each other is that $\Phi_{l} g_{j i}=0$, where $\Phi$ is the operator associated to $\varphi_{i}{ }^{j}=\varphi_{i r} g^{r_{j}}$.

In the rest of the present section, we shall assume that $g_{j i}$ is a $\Phi$-tensor, and denote by $\nabla_{i}$ the operator of the Riemannian covariant derivative with respect to $g_{j i}$. From Theorem 2, we know that $\Phi_{l} g_{j i}=0$ is equivalent to $\nabla_{k} \varphi_{j i}=0$.

Let $R_{k j i}{ }^{h}$ and $S_{k j i}{ }^{h}$ be the Riemannian curvature tensors formed by $g_{j i}$ and $\boldsymbol{\varphi}_{j i}$ respectively, then we have $R_{k j i}{ }^{h}=S_{k j i}{ }^{h}$ by means of the assumption.

Applying the Ricci's identity to $\boldsymbol{\varphi}_{i}{ }^{h}$, we get $R_{k j ; r}{ }^{n} \boldsymbol{\varphi}_{i}{ }^{r}=R_{k j i}{ }^{r} \boldsymbol{\varphi}_{r}{ }^{h}$, which shows that $R_{k j i}{ }^{h}$ is pure with respect to $i$ and $h$. Hence $R_{k j i h}=R_{k j i}{ }^{r} g_{r \cdot h}$ is pure with respect to $k$ and $j$ and also pure with respect to $i$ and $h$.

On the other hand, $S_{k j i}{ }^{h}$ being the Riemannian curvature tensor formed by $\boldsymbol{\phi}_{i i}$, if we put $T_{k j i h}=S_{k j i}{ }^{r} \boldsymbol{\varphi}_{r h}$, then we have

$$
T_{k j i k}=T_{i h k j} \text {. }
$$

Since it holds that $T_{k j i h}=R_{k j i r} \boldsymbol{\varphi}_{h}{ }^{r}$ and $T_{i h k j}=R_{k r i h} \boldsymbol{\varphi}_{j}^{r}$, the equation (15) 
becomes $R_{k j i r r} \boldsymbol{\varphi}_{h}{ }^{r}=R_{k r i h} \boldsymbol{\varphi}_{j}{ }^{r}$, which shows that $R_{k j i h}$ is pure with respect to $j$ and $h$. Therefore $R_{k j i h}$ is pure.

Since we have

$$
\begin{aligned}
\Phi_{l} R_{k j i h} & =\varphi_{l}^{r} \nabla_{r} R_{k j i h}-\nabla_{l} R_{k j i h}^{*} \\
& =-\varphi_{l}^{r}\left(\nabla_{k} R_{j r i h}+\nabla_{j} R_{r k i h}\right)-\varphi_{h}^{r} \nabla_{l} R_{k j i r} \\
& =-\varphi_{h}^{r}\left(\nabla_{k} R_{j l i r}+\nabla_{j} R_{l k i r}+\nabla_{l} R_{k j i r}\right)=0,
\end{aligned}
$$

$\nabla \iota R_{k j i h}$ is also pure.

LEMMA 7. Let us assume that $\Phi_{l} g_{j i}=0$. If a tensor, say $T$, and its covariant derivative are pure, then we have

$$
\nabla_{t} \Phi_{l} T=\Phi_{l} \nabla_{t} T
$$

PROOF. Let $T$ be a tensor of type $(1,1)$, for example. Then we have

$$
\nabla_{t} \Phi_{l} T_{i}{ }^{j}-\Phi_{l} \nabla_{t} T_{i}{ }^{j}=\varphi_{l}{ }^{r}\left(\nabla_{t} \nabla_{r}-\nabla_{r} \nabla_{t}\right) T_{i}{ }^{j}-\left(\nabla_{t} \nabla_{l}-\nabla_{l} \nabla_{t}\right) \stackrel{*}{T_{i}}{ }^{j}
$$

On the other hand, it holds that

$$
\begin{aligned}
& \left(\nabla_{t} \nabla_{l}-\nabla_{l} \nabla_{t}\right) \stackrel{{ }^{*}}{T_{i}}{ }^{j}=R_{t l r}{ }^{j}{ }^{*} T_{i}^{r}-R_{t l i}{ }^{r} \stackrel{*}{T}_{r}{ }^{j} \\
& =R_{t l r}{ }^{j} \varphi_{s}^{r} T_{i}^{s}-R_{t l i}{ }^{r} \varphi_{r}{ }^{s} T_{s}{ }^{j} \\
& =R_{t r s}{ }^{j} \varphi_{l}^{r} T_{i}^{s}-R_{t r i}{ }^{s} \varphi_{l}^{r} T_{s}^{j} \\
& =\varphi_{l}{ }^{r}\left(\nabla_{t} \nabla r-\nabla r \nabla_{t}\right) T_{i}{ }^{j} .
\end{aligned}
$$

From these equations, we find that the lemma is true.

q. e. d.

If we apply Lemma 7 to our $R_{k j i}{ }^{h}$, then we have $\Phi_{l} \nabla_{t} R_{k j i h}=0$, which shows that $\nabla_{l} \nabla_{t} R_{k j i n}$ is pure. Thus we get

THEOREM 3. Let $g_{j i}$ and $\boldsymbol{\varphi}_{j i}$ be two Riemannian metrics and $\Phi$ be the operator associated to ${\phi_{i}}^{j}=\phi_{i r} g^{r \cdot 1}$. If $\Phi_{l} g_{j i}=0$ is valid, then $R_{k j i n}$ and its successive covariant derivatives are pure.

Let $\varphi_{i}{ }^{j}$ be an almost-product structure. then there exists a Riemannian metric $g_{j i}$ such that $g_{r s} \boldsymbol{\varphi}_{j}{ }^{r} \boldsymbol{\varphi}_{i}{ }^{s}=g_{j i}$. Then we know that the tensor $\boldsymbol{\phi}_{j i}=\boldsymbol{\phi}_{j}{ }^{r}$ $g_{r \cdot i}$ is also a Riemannian metric. Thus theorems in this section are applicable to this case.

7. In this section we shall assume that ${\varphi_{i}}^{r} \boldsymbol{\varphi}_{r}{ }^{j}=\varepsilon \delta_{i}{ }^{j}$.

If we put $\Phi_{l} \boldsymbol{\varphi}_{i}{ }^{j}=N_{l i}{ }^{j}$, then it holds that

$$
\begin{aligned}
N_{l i}{ }^{j} & =\boldsymbol{\varphi}_{l}{ }^{r} \partial_{r} \boldsymbol{\varphi}_{i}{ }^{j}+\left(\partial_{i} \boldsymbol{\varphi}_{l}{ }^{r}\right) \boldsymbol{\varphi}_{r}{ }^{j}+\left(\partial_{l} \boldsymbol{\varphi}_{r}{ }^{j}-\partial_{r} \boldsymbol{\varphi}_{l}{ }^{j}\right) \boldsymbol{\varphi}_{i}{ }^{r} \\
& =\boldsymbol{\varphi}_{l}{ }^{r}\left(\partial_{r} \boldsymbol{\varphi}_{i}{ }^{j}-\partial_{i} \boldsymbol{\varphi}_{r}{ }^{j}\right)-\boldsymbol{\varphi}_{i}{ }^{r}\left(\partial_{r} \boldsymbol{\varphi}_{l}{ }^{j}-\partial_{l} \boldsymbol{\varphi}_{r}{ }^{j}\right),
\end{aligned}
$$


which is nothing but the Nijenhuis' tensor $[1],[3],[4],[10],[11],[12]$. It satisfies the equations

$$
N_{l i}{ }^{j}=-N_{i l}{ }^{j}, \quad N_{l i}{ }^{j} \boldsymbol{Q}_{i}{ }^{r}=-N_{l i}{ }^{r} \boldsymbol{\phi}_{r}{ }^{j} .
$$

The last equation shows that $N_{l i}{ }^{j}$ is hybrid with respect to $i$ and $j$, hence takıng account of the skew-symmetricity of $N_{l i}{ }^{j}$, it is pure with respect to $i$ and $l$. Thus we get

$$
N_{l r}{ }^{j} \boldsymbol{\phi}_{i}{ }^{r}=N_{r i}{ }^{j} \boldsymbol{\phi}_{l}{ }^{r}, \quad N_{l r}{ }^{r}=0, \quad N_{l r}{ }^{t} \boldsymbol{\phi}_{l}{ }^{r}=0,
$$

by virtue of Lemma 4 and Lemma 5 .

Now we introduce an affine connection $\Gamma_{j i}^{h}$ such that

$$
\nabla_{\imath} \varphi_{i}{ }^{j}=0, \quad S_{j i}{ }^{h}=-(\varepsilon / 8) N_{j i}{ }^{n},
$$

where $\nabla_{l}$ denotes the operator of the covariant derivative with respect to $\Gamma_{j i}^{h}$ and $S_{j i}{ }^{h}$ its torsion tensor.

It is known that there exists such a connection, which will be called the canonical connection [12].

If we make use of the canonical connection, the equation (10) becomes

$$
\Phi_{l} \xi_{(i)}^{(j)}=\phi_{l}^{r} \nabla r \xi_{(i)}^{(j)}-\nabla_{l} \stackrel{*}{(i)}^{(j)}
$$

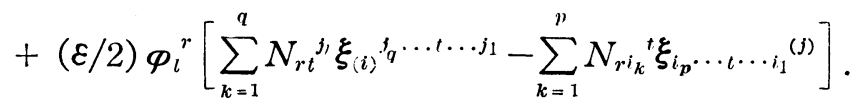

Making use of the form (16), we shall obtain some formulas on the operator $\Phi$.

The tensor $\boldsymbol{\varphi}_{i}{ }^{j}$ being pure, if we substitute it in the place of $\xi$ or $\eta$ in (14), then we get the following formulas.

$$
\begin{aligned}
& \Phi_{l}{\stackrel{*}{\xi_{(i)}}}^{b(j)}=\phi_{r}{ }^{b} \Phi_{l} \xi_{(i)}{ }^{r(j)}+N_{l r}{ }^{b} \xi_{(i)}{ }^{r(j)}, \\
& \Phi_{l}{\stackrel{\xi}{\xi_{a(i)}}}^{(j)}={\phi_{a}}^{r} \Phi_{l} \xi_{r(i)}{ }^{(j)}+N_{l a}{ }^{r} \xi_{r(i)}{ }^{(j)} .
\end{aligned}
$$

We can see also that

$$
\begin{aligned}
& \Phi_{l} \stackrel{*}{\xi}(i)^{(j)}=\phi_{r}^{j_{i}} \Phi_{l} \xi_{(i)}{ }^{j_{q} \cdots r \cdots j_{1}}+N_{l r}{ }^{j_{k}} \xi_{(i)}{ }^{j_{1} \cdots r \cdots j_{1}} \text {, if } q \geqq 1 \text {, }
\end{aligned}
$$

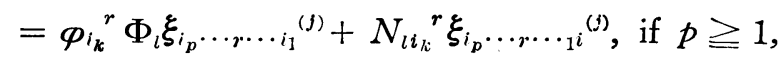

are valid.

In the next place, we shall prove the following formula :

$$
\Phi_{l} \stackrel{*}{\xi}_{(i)}^{(1)}=-\phi_{l}^{r} \Phi_{r} \dot{\xi}_{(i)}^{(j)}+\sum_{k=1}^{q} N_{l r}{ }^{j}{ }^{k} \xi_{(l)}{ }^{j} q^{\ldots \ldots r \ldots s_{1}} .
$$


In fact, we have

$$
\begin{aligned}
& \Phi_{l} \stackrel{*}{\xi}_{(i)}^{(j)}=\varphi_{l}^{r} \nabla_{r} \stackrel{*}{\xi}_{(i)}^{(j)}-\varepsilon \nabla_{l} \xi_{(i)}^{(1)}
\end{aligned}
$$

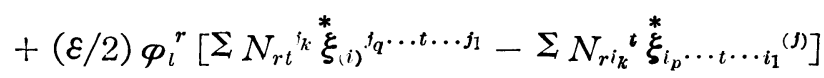

$$
\begin{aligned}
& =-\varepsilon \nabla_{l} \xi_{(i)}^{(j)}+\varphi_{l}^{r} \nabla_{r} \stackrel{\xi}{\xi}_{(i)}^{(j)}
\end{aligned}
$$

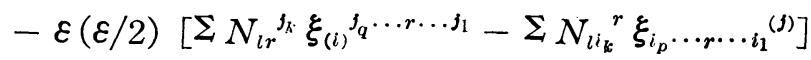

$$
\begin{aligned}
& +\sum N_{l r}{ }^{j_{i}} \xi_{(i)}{ }^{i}{ }^{\prime} \ldots r \cdots j_{1} \\
& =-\varphi_{l}{ }^{r} \Phi_{r} \xi_{(i)}^{(j)}+\sum N_{l r}{ }^{j_{k}} \xi_{(i)}{ }^{j}{ }^{j} \cdots r \cdots \gamma_{1} \text {. q. e. d. }
\end{aligned}
$$

Especially, for a pure tensor $\xi_{(i)}$ of type $(0, p)$, we have

$$
\Phi_{l} \stackrel{*}{\xi}_{(i)}=-\varphi_{i}^{r} \Phi_{r} \xi_{(i)},
$$

from which it holds, taking account of (14),

$$
N_{l t}^{r} \xi_{r(i)}+\varphi_{t}^{r} \Phi_{l} \xi_{r(i)}=-\varphi_{l}^{r} \Phi_{r} \xi_{t(i)} .
$$

From (18) we have

THEOREM 4. Let $\phi_{i}{ }^{j}$ satisfies ${\varphi_{i}}^{r} \phi_{r}{ }^{j}=\varepsilon \delta_{i}{ }^{j}$. If $\xi_{(i)}$ is a $\Phi$-tensor, then so is $\stackrel{*}{\xi}_{(i)}^{*}$.

In this case, we know by virtue of (19) that the relation

$$
N_{l j}^{r} \xi_{i_{p} \cdots r i_{1}}=0
$$

holds good.

Next we shall generalize the fact that $\Phi_{l} \varphi_{i}{ }^{j}=N_{l i}{ }^{3}$ is hybrid with respect to $l$ and $j$.

THEOREM 5. Let $\boldsymbol{\varphi}_{i}{ }^{j}$ satisfies $\boldsymbol{\varphi}_{i}{ }^{r} \varphi_{r}{ }^{j}=\varepsilon \delta_{i}{ }^{j}$. If $\xi_{(i)}{ }^{j}$ is a pure tensor of type $(1, p)$, then $\Phi_{l} \xi_{(i)}{ }^{j}$ is hybrid with respect to $l$ and $j$.

In fact, we have by virtue of (17)

$$
\Phi_{l}^{*} \stackrel{\xi}{\xi}_{(i)}^{j}=-\varphi_{l}^{r} \Phi_{r} \xi_{(i)}^{j}+N_{l r}^{j} \xi_{(i)}^{r} .
$$

On the other hand, taking account of (14), ws find that

$$
\Phi_{l}^{*} \xi_{(i)}^{j}=\Phi_{l}\left(\xi_{(i)}^{r} \varphi_{r}^{j}\right)=\varphi_{r}^{j} \Phi_{l} \xi_{(i)}^{r}+N_{l r}{ }^{j} \xi_{(i)}^{r} \text {. }
$$

From these equations we obtain the theorem.

q. e. d.

If we define $A_{l k j i}{ }^{h}=N_{l k}{ }^{a} N_{j i}{ }^{b} N_{a b}{ }^{h}$, then it is evidently a pure tensor of type $(1,4)$, hence $\Phi_{t} A_{l i k j i}{ }^{h}$ is a tensor which is hybrid with respect to $t$ and 
$h$. It depends only on $\boldsymbol{\varphi}_{i}{ }^{j}$ and contains its second derivatives. From Lemma 4, we have $\Phi_{r} A_{l k j i}{ }^{r}=0$, which may be a new identity on $\boldsymbol{\varphi}_{i}{ }^{j}$.

We denote by $C$ the contraction's operator, i. e., if $C$ means the contraction with respect to $i_{1}$ and $j_{1}$, for example, then $C \xi_{(i)}{ }^{(j)}=\xi_{i_{p}} \cdots i_{2} r^{j_{q} \cdots j_{2} r}$. If $\xi_{(i)}{ }^{(j)}$ is a pure tensor of type $(q, p)$, then the tensor $C \xi_{(i)}^{(j)}$ is also pure if it is not a scalar. Making use of (16), we can verify the following relation, after some calculations.

$$
C \Phi_{l} \xi_{(i)}^{(j)}=\Phi_{l} C \xi_{(i)}^{(j)} \text {. }
$$

In (20), we assumed that $C \xi_{(i)}{ }^{(j)}$ is not a scalar and $C$ operates on the same indices of both sides.

From (20) we have

THEOREM 6. Let $\boldsymbol{\varphi}_{i}{ }^{j}$ satisfies ${\varphi_{i}}^{r} \varphi_{r}{ }^{j}=\varepsilon \delta_{i}{ }^{j}$. If $\xi_{(i)}{ }^{(j)}$ is a Ф-iensor, then so is $C \xi_{(i)}^{(i)}$ provided that it is not a scalar.

Let $\xi_{(i)}^{(i)}$ and $\eta_{(i)}{ }^{(i)}$ be pure tensors of type $(q, p)$ and type $(p, q)$ respectively. Then we have

$$
\eta_{(j)}^{(i)} \Phi_{l} \xi_{(i)}^{(j)}=\eta_{(j)}^{(i)} \varphi_{l}^{r} \nabla_{r} \xi_{(i)}^{(j)}-\eta_{(j)}^{(i)} \nabla_{l} \xi_{(i)}^{*},
$$

because we have from Lemma 4 and the hybridity of $N_{l i}{ }^{j}$,

$$
N_{r i_{k}}{ }^{t} \xi_{i_{p}} \cdots_{t} \cdots i_{1}{ }^{(j)} \eta_{(j)}{ }^{(i)}=0 .
$$

In the same manner, we get

$$
\begin{aligned}
\xi_{(i)}^{(j)} \Phi_{l} \eta_{(j)}{ }^{(i)} & =\xi_{(i)}^{(j)} \Phi_{l}^{r} \nabla_{r} \eta_{(j)}^{(i)}-\xi_{(i)}^{(j)} \nabla_{l}{ }^{*}{ }_{(j)}^{(i)} \\
& =\xi_{(i)}^{(j)} \Phi_{l}{ }^{r} \nabla_{r} \eta_{(j)}{ }^{(i)}-\stackrel{\xi}{\xi}_{(i)}^{(j)} \nabla_{l} \eta_{(j)}^{(i)}
\end{aligned}
$$

Hence we obtain

$$
\xi_{(i)}^{(j)} \Phi_{l} \eta_{(j)}^{(i)}+\eta_{(i)}^{(i)} \Phi_{l} \xi_{(i)}^{(j)}=\phi_{l}^{r} \partial_{r}\left(\xi_{(i)}^{(i)} \eta_{(i)}^{(j)}\right)-\partial_{l}\left(\xi_{(i)}^{*}{ }^{(j)} \eta_{(j)}^{(i)}\right)
$$

8. Let us consider an almost-Hermitian space $M$ whose positive definite Riemannian metric is $g_{j i}$ and the almost-complex structure is $\boldsymbol{\varphi}_{i}{ }^{j}$. By definition these tensors satisfy $g_{r s} \boldsymbol{\varphi}_{j}^{r} \boldsymbol{\varphi}_{i}{ }^{s}=g_{j i}$, from which $\boldsymbol{\Phi}_{j i}={\boldsymbol{P}_{j}}^{r} g_{r i}$ is skewsymmetric. Now we assume that $\nabla_{r} \boldsymbol{\varphi}_{i}{ }^{r}=0$, where $\nabla r$ denotes the operator of the Riemannian covariant derivative. The following lemma is known [7], $[8]$.

LEMMA 8. Let $M$ be a compact almost-Hermitian space satisfying $\nabla r \boldsymbol{\phi}_{i}{ }^{r}=0$. If scalar functions $f$ and $g$ satisfy $\partial_{i} f=\phi_{i}{ }^{r} \partial_{r} g$, then they are both constant over $M$.

From this lemma and (21) we have 
THEOREM 7. Let $M$ be a compact almost-Hermitian space satisfying $\nabla, \varphi_{i}{ }^{r}=0$. If $\xi_{(i)}^{(j)}$ and $\eta_{(j)}{ }^{(i)}$ are $\Phi$-tensors of type $(q, p)$ and of type $(p, q)$ respectively, then the inner product $\xi_{(l)}^{(j)} \eta_{(j)}^{(1)}$ is constant.

COROLLARY. Let $M$ be a compact almost-Hermitian space satisfying $\nabla r \varphi_{i}{ }^{r}=0$. If $\xi_{(1)}^{(j)}$ is a $\Phi$-tensor of type $(q, p)$ and $v^{i}(\alpha=1, \ldots \ldots, p),{ }_{u}^{a}(\alpha=1$,

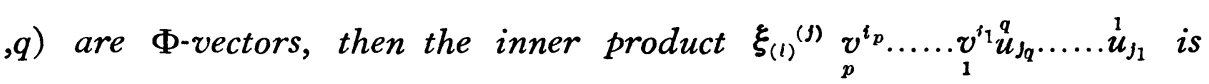
constant.

9. Let us consider a Kählerian space $M$ with a positive definite metric. We shall make use of the notation in $\S 2$.

An analytic tensor $\xi_{(i)}^{(j)}$ is by definition a pure tensor such that $\nabla_{l} \xi_{(i)}^{(j)}$ is also pure.

Now we define, for a pure tensor $\xi_{(i)}^{(j)}$,

$$
\begin{aligned}
a_{k(i)}{ }^{(j)}(\xi) & =\nabla_{k} \xi_{(i)}{ }^{(j)}+{\phi_{k}}^{l}{\phi_{i}}^{r} \nabla_{l} \xi_{i p} \ldots i_{2 r}{ }^{(j)} \\
& =\nabla_{k} \xi_{(i)}{ }^{(j)}+\phi_{k}{ }^{l} \phi_{r}{ }^{j_{1}} \nabla_{l} \xi_{(i)}{ }^{j a \ldots j_{2} r} .
\end{aligned}
$$

$a_{k(i)}^{(j)}(\xi)=0$ is equivalent to that the pure tensor $\xi_{(i)}^{(j)}$ is analytic.

On taking account of that the Riemannian curvature tensor $R_{k j i}{ }^{k}$ and Ricci tensor $R_{j i}$ of a Kählerian space satisfy

$$
\text { - (1/2) } \boldsymbol{\phi}^{l t} R_{l t i}{ }^{h}=R_{i}{ }^{r} \boldsymbol{\phi}_{r}{ }^{h}=R_{r}{ }^{h} \boldsymbol{\phi}_{i}{ }^{r},
$$

we can easily obtain

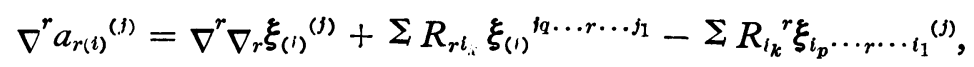

where $\nabla^{r}=g^{r t} \nabla_{t}$. Hence if $\xi_{(t)}^{(j)}$ is analytic, then it satisfies

$$
\nabla^{r} \nabla r \xi_{(i)}{ }^{(j)}+\Sigma R_{r}{ }^{j} \xi_{(i)}{ }^{j}{ }^{\prime} \cdots r \cdots s_{1}-\Sigma R_{i_{k}}{ }^{r} \xi_{i_{p} \cdots \cdots_{1}}{ }^{(j)}=0 .
$$

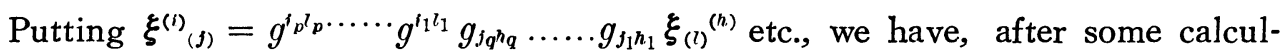
ations,

$$
\nabla^{r}\left(a_{r(i)}^{(j)} \xi^{(i)}(j)\right)=\left(\nabla^{r} a_{r(i)}^{(j)}\right) \xi^{(i)}{ }_{(j)}+(1 / 2) a^{2}(\xi),
$$

where

$$
a^{2}(\xi)=a_{r(i)}^{(j)} a^{r(i)}(j)
$$

Thus, by Green's theorem, we have

THEOREM 8.5) In a compact Kählerian space $M$, the integral formula

5) For a skew-symmetric contravariant pure tensor, see [6]. 


$$
\begin{array}{r}
\int_{M r}\left[\left(\nabla^{r} \nabla r \xi_{(i)}^{(j)}+\sum_{k=1}^{q} R_{r}^{{ }^{{ }_{k}} \xi_{(i)}{ }^{j q \cdots r \cdots j_{1}}}-\sum_{k=1}^{p} R_{i_{k}}{ }^{r} \xi_{i_{p}} \cdots \boldsymbol{r} \cdots i_{1}{ }^{(\boldsymbol{g})}\right) \xi^{(i)}(\mathrm{J})\right. \\
\left.+(1 / 2) a^{2}(\xi)\right] d \sigma=0
\end{array}
$$

is valid for a pure tensor $\xi_{(i)}^{(j)}$, where $d \sigma$ is the volume element of $M$ and $a^{2}(\xi)$ is given by (22).

THEOREM 9. In a compact Kählerian space, a necessary and sufficient condition for a pure tensor $\xi_{(i)}^{(j)}$ to be analytic is that it satisfies

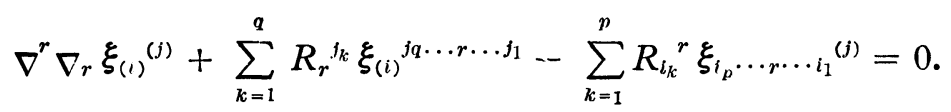

On the other hand, in a compact orientable Riemannian space, a necessary and sufficient condition for a skew-symmetic tensor $\xi_{(i)}$ to be harmonic is that [13]

$$
\nabla^{r} \nabla_{r} \xi_{(i)}-\sum R_{i_{k}}{ }^{r} \xi_{i_{p} \cdots r \cdots i_{1}}+\sum_{l>k} R_{i_{l} i_{k}{ }^{r s}} \xi_{l_{p} \cdots r \cdots s} \cdots_{i_{1}}=0 .
$$

Let $\xi_{(i)}$ be a skew-symmetric pure tensor, then

$$
R_{k j}^{r s} \xi_{i_{p}} \cdots_{r} \cdots \cdots_{i} \cdots_{1}=0,
$$

by vritue of Lemma 5 and the hybridity of $R_{k j}{ }^{r s}$ with respect to $r$ and $s$. Thus we have

COROLLARY [13]. In a compact Kählerian space, a necessary and sufficient condition for a skew-symmetric pure tensor to be analytic is that it is harmonic.

If $\xi_{(\mathfrak{})}$ is skew-symmetric pure tensor, then so is $\stackrel{\xi}{(i)}^{*}$ by virtue of Lemma 2. Hence taking account of Theorem 4, in a compact Kählerian space, if a pure tensor $\xi_{(i)}$ is harmonic, then so is $\stackrel{*}{\xi}_{(i)}$.

\section{BIBLIOGRAPHY}

[1] LEgrand, G., Sur les varieté à structure de presque-produit complexe, C. R. Paris (1956), 335-337.

[2] LEgRAND, G., Structure presque hermitiennes au sens large, C. R. Paris (1956), 13921395.

[3] Nijenhuis, A., $X_{2 n}$-forming sets of eigenvectors, Indag. Math. 13 (1951), 200-212.

[4] OBATA, M., Affine connections on manifolds with almost complex, quaternion or Hermitian structure, Japanese Jour. of Math. 26(1956), 43-77.

[5] SASAKI, S. AND YANO, K., Pseudo-analytic vectors on pseudo-Kählerian manifolds, Pacific. Jour. 5(1955), 987-993. 
[6] SAWAKI, S. AND Kото̄, S., On the analytic tensor in a compact Kaehler space, Jour. of the faculty of Sci. Niigata Univ. (1958), 77-84.

[7] TACHIBANA, S., On almost-analytic vectors in almost-Kählerian manifolds, Tôhoku Math. Jour. 11(1959), 247-265.

[8] TACHIBANA, S., On almost-analytic vectors in certain almost-Hermitian manifolds, Tôhoku Math. Jour. 11 (1959), 351-363

[9] Walker, A. G., Connexions for parallel distributions in the large, Quarterly Jour. (1955), 301-308.

[10] WALKER, A. G., Dérivation torsionnelle et seconde torsion pour une structure presquecomplexe, C. R. Paris (1957), 1213-1215.

[11] YANO, K., The theory of Lie derivatives and its applications, Amsterdam, (1957).

[12] YANC, K., On Walker differentiation in almost product or almost complex spaces, Indag. Math. 20(1958), 573-580.

[13] YANO, K. AND BOCHNER, S., Curvature and Betti numbers, Annals of Math. Studies, No. 32, (1953).

OCHANOMIZU UNIVERSITY, TOKYO. 